\title{
Existence and nonexistence of solutions for quasilinear elliptic systems
}

\author{
Lei Wei and Zhaosheng Feng
}

Communicated by $Y$. Charles Li, received November 26, 2012.

\begin{abstract}
In this paper, we are concerned with positive solutions of quasilinear elliptic systems with the homogeneous Dirichlet boundary condition. In the case of $\alpha \beta>(p-1)(q-1)$, under certain conditions we show that there exists $\lambda^{*}>0$ such that for any $\lambda \in\left(0, \lambda^{*}\right)$ the boundary value problem has at least two positive solutions. In the case of $\alpha \beta<(p-1)(q-1)$, we establish a priori estimates and the existence of positive solutions.
\end{abstract}

\section{Contents}

1. Introduction 25

2. Preliminaries 29

3. Proof of Theorem $1 \quad 30$

4. Proofs of Theorems 2 and $3 \quad 35$

Acknowledgments $\quad 41$

References $\quad 41$

\section{Introduction}

Elliptic equations or systems, any of a class of partial differential equations describes phenomena that do not change from moment to moment, as when a flow of heat or fluid takes place within a medium with no accumulations. In addition to satisfying a differential equation within the region, the elliptic equation is also determined by its values (boundary values) along the boundary of the region, which represent the effect from outside the region. These condition can be either those of a fixed temperature distribution at points of the boundary (Dirichlet problem) or

1991 Mathematics Subject Classification. 35J62, 35B45, 35D30.

Key words and phrases. Eigenvalue, positive solutions, topological degree, quasilinear elliptic system, a priori estimates.

This work is supported by Natural Science Foundation of Jiangsu Province under 11KJB110015 and Tianyuan Natural Science Foundation under 11126344. 
those in which heat is being supplied or removed across the boundary in such a way as to maintain a constant temperature distribution throughout (Neumann problem) $[\mathbf{3}, \mathbf{1 7}]$. Nowadays, elliptic partial differential equations have applications in almost all areas of mathematics, from harmonic analysis to geometry to Lie theory, as well as numerous applications in physics and engineering with a well-developed theory $[2,4,5,7,9,13,19,20,21]$.

Consider the quasilinear elliptic system

$$
\begin{cases}-\Delta_{p} u=\lambda\left(a+u^{\delta}+v^{\alpha}\right), & x \in \Omega, \\ -\Delta_{q} v=\lambda\left(b+u^{\beta}+v^{\gamma}\right), & x \in \Omega, \\ u>0, \quad v>0, & x \in \Omega \\ u=v=0, & x \in \partial \Omega\end{cases}
$$

where $a$ and $b$ are two positive constants, and $\Omega$ is a bounded smooth domain. One can see that system (1.1) has no variational structure. So it is difficult to study the existence of the solution of system (1.1) by using variational methods. For certain non-variational quasilinear elliptic systems, the existence of nontrivial solutions has been studied extensively $[\mathbf{2}, \mathbf{5}, \mathbf{1 9}, \mathbf{2 0}]$.

Let us briefly review some related studies on the topic. In [5], Clément et al considered positive radial solutions of the quasilinear elliptic system

$$
\begin{cases}-\Delta_{p} u=u^{\alpha} v^{\beta}, & x \in B_{R}, \\ -\Delta_{q} v=u^{\gamma} v^{\delta}, & x \in B_{R}, \\ u>0, \quad v>0, & x \in B_{R}, \\ u=v=0, & x \in \partial B_{R},\end{cases}
$$

and assumed the so-called globally superhomogeneous structure

$$
\beta \gamma>(p-1-\alpha)(q-1-\delta)
$$

System (1.2) is unstable in the sense that possible solutions of (1.2) cannot be obtained by iterative methods or the upper-lower solutions technique. A sufficient condition was obtained for the existence of a positive radial solution of system (1.2) in the nonvariational case.

In [2], Azizieh et al used continuation and moving hyperplane methods to prove existence and a priori estimates for $p$-Laplace systems of the form

$$
\begin{cases}-\Delta_{p_{1}} u=f(|v|), & x \in \Omega, \\ -\Delta_{p_{2}} v=g(|u|), & x \in \Omega, \\ u=v=0, & x \in \partial \Omega,\end{cases}
$$

where $1<p_{1}, p_{2}<N, \Omega \subset \mathbb{R}^{N}$ is bounded and convex, and $f, g: \mathbb{R} \rightarrow \mathbb{R}^{+}$are nondecreasing, locally Lipschitz continuous functions satisfying

$$
C_{1}|s|^{q_{1}} \leq f(s) \leq C_{2}|s|^{q_{1}}, \quad D_{1}|s|^{q_{2}} \leq g(s) \leq D_{2}|s|^{q_{2}}, \quad \forall s \in \mathbb{R}^{+}
$$

for some positive constants $C_{1}, C_{2}, D_{1}$ and $D_{2}$, and $q_{1} q_{2}>\left(p_{1}-1\right)\left(p_{2}-1\right)$. 
In [19], Wang dealt with the existence and nonexistence of positive radial solutions for the weakly coupled quasilinear system

$$
\left\{\begin{aligned}
-\Delta_{p} u & =\lambda f(v), & & x \in B_{1}(0), \\
-\Delta_{p} v & =\lambda g(u), & & x \in B_{1}(0), \\
u=v & =0, & & x \in \partial B_{1}(0) .
\end{aligned}\right.
$$

It is shown that there is a positive radial solution of the problem for various intervals of $\lambda$ in sublinear cases by the topological degree theory and fixed point theorems in a cone.

We denote

$$
W=W_{0}^{1, p}(\Omega) \times W_{0}^{1, q}(\Omega), \quad E=C_{0}^{1}(\bar{\Omega}) \times C_{0}^{1}(\bar{\Omega}) .
$$

$B_{R}$ is the ball in $\mathcal{R}^{N}(N>1)$ with center zero and radius $R$. A connected and closed set $S^{+} \subset[0, \infty) \times E$ is said to be a continuum of solutions of system (1.1) if $S^{+}$ consists of solutions $(\lambda, u, v)$ of system (1.1). Generally, $(u, v) \in W_{0}^{1, p}(\Omega) \times W_{0}^{1, q}(\Omega)$ is said to be a weak solution of system (1.1) if for any $\left(\phi_{1}, \phi_{2}\right) \in W_{0}^{1, p}(\Omega) \times W_{0}^{1, q}(\Omega)$ we have

$$
\begin{aligned}
& \int_{\Omega}|\nabla u|^{p-1} \nabla u \nabla \phi_{1}=\lambda \int_{\Omega}\left(a+u^{\delta}+v^{\alpha}\right) \phi_{1}, \\
& \int_{\Omega}|\nabla v|^{q-1} \nabla v \nabla \phi_{2}=\lambda \int_{\Omega}\left(b+u^{\beta}+v^{\gamma}\right) \phi_{2} .
\end{aligned}
$$

Note that if $1<p$ and $q<N$, then it has

$$
W_{0}^{1, p}(\Omega) \times W_{0}^{1, q}(\Omega) \hookrightarrow L^{\infty}(\Omega) \times L^{\infty}(\Omega) .
$$

Since

$$
\left(-\Delta_{p}\right)^{-1}: L^{\infty}(\Omega) \rightarrow C_{0}^{1+\eta}(\bar{\Omega}), \quad\left(-\Delta_{q}\right)^{-1}: L^{\infty}(\Omega) \rightarrow C_{0}^{1+\eta}(\bar{\Omega})
$$

are completely continuous $(0<\eta<1)[\mathbf{1 8}]$, any weak solution $(u, v)$ of system $(1.1)$ is a solution belonging to $C_{0}^{1+\eta}(\bar{\Omega}) \times C_{0}^{1+\eta}(\bar{\Omega})$, namely, $(u, v) \in E$. But in general when $p, q>1$, the weak solution of system (1.1) may not necessarily belong to $E$. Throughout this paper, if there is no special statement, a solution of system (1.1) is considered to belong to $E$ or $C_{0}^{1+\eta}(\bar{\Omega}) \times C_{0}^{1+\eta}(\bar{\Omega})$.

In [20], the regularity (or partial regularity) of weak solutions to a quasilinear elliptic system with lower-order terms was studied. The main aim of this paper is to use topological methods to study the existence and nonexistence of solutions of system (1.1) in the cases of $\alpha \beta>(p-1)(q-1)$ and $\alpha \beta<(p-1)(q-1)$, respectively. Under the conditions that $\alpha \beta>(p-1)(q-1)$ and $\Omega$ is a ball, we will establish a priori estimates of solutions by means of the blow-up method. Furthermore, we obtain the following Theorem 1 by using the topological degree theory. For a single equation, the similar problems have been studied in $[\mathbf{1 0}, \mathbf{1 4}]$. For example, in $[\mathbf{1 0}]$, Fleckinger and Reichel were concerned with global continua of positive solutions for the equation

$$
\begin{cases}-\Delta_{p} u=\lambda\left(1+u^{q}\right), & x \in \Omega, \\ u=0, & x \in \partial \Omega,\end{cases}
$$

where $q>p-1$ and $1<p \leq 2$. By using a topological degree argument and a weighted Poincaré inequality, they obtained that there exists an unbounded continuum of solutions for subcritical values of $q$. For supercritical exponents $q$ it is shown that the problem has a unique solution for the small values of $\lambda>0$. 
Motivated by these results, we obtain the following Theorem on an unbounded continuum of solutions of system (1.1):

THEOREM 1. Suppose that $\Omega=B_{R}$. Let

$$
\begin{gathered}
\alpha \beta>(p-1)(q-1), \quad 1<p, q<N, \\
\max \left\{N_{1}-\frac{N-p}{p-1}, N_{2}-\frac{N-q}{q-1}\right\} \geq 0
\end{gathered}
$$

and

$$
p-1 \leq \delta<(p-1)+p / N_{1}, \quad q-1 \leq \gamma<(q-1)+q / N_{2}
$$

where

$$
N_{1}=\frac{q \alpha+p(q-1)}{\alpha \beta-(p-1)(q-1)}, \quad N_{2}=\frac{p \beta+q(p-1)}{\alpha \beta-(p-1)(q-1)} .
$$

Then, the following assertions hold.

(a) There exists an unbounded continuum $S^{+} \subset[0, \infty) \times C_{0}^{1}(\bar{\Omega}) \times C_{0}^{1}(\bar{\Omega})$ of solutions of system (1.1), which is bounded in the $\lambda$-direction.

(b) There exists a positive value $\lambda^{*}>0$ such that

(i) for any $0<\lambda<\lambda^{*}$, system (1.1) has at least two solutions on $S^{+}$;

(ii) for $\lambda=\lambda^{*}$, system (1.1) has at least one solution on $S^{+}$;

(iii) for any $\lambda>\lambda^{*}$, system (1.1) has no solution.

When $\alpha \beta<(p-1)(q-1)$ and $\Omega$ is a bounded smooth domain, we do not make use of the blow-up method, since it is unclear whether there exists the corresponding Liouville-type theorem in this case.

Let

Assume that

$$
p^{*}=\frac{n p}{n-p}, \quad q^{*}=\frac{n q}{n-q} .
$$

$\left(H_{1}\right) \quad 0<\delta<p-1$ and $0<\gamma<q-1$;

$\left(H_{2}\right) \frac{p}{p^{*}}+\frac{\alpha}{q^{*}}<1$ and $\frac{q}{q^{*}}+\frac{\beta}{p^{*}}<1$.

Under conditions $\left(H_{1}\right)$ and $\left(H_{2}\right)$, we consider a priori estimates of weak solutions of system (1.1) by the regularity arguments. Moreover, we have the following result:

TheOREM 2. Suppose that $\alpha \beta<(p-1)(q-1)$, and conditions $\left(H_{1}\right)$ and $\left(H_{2}\right)$ hold. Then for any $\lambda>0$, system (1.1) has at least one solution.

When $\delta=p-1$ and $\gamma=q-1$ in system (1.1), we have the following theorem:

THEOREM 3. Let $\delta=p-1$ and $\gamma=q-1$ in system (1.1). Suppose that $\alpha \beta<(p-1)(q-1)$ and $\left(H_{2}\right)$ holds. Then there exists $\lambda^{*}>0$ such that

(i) system (1.1) has no solution for any $\lambda>\lambda^{*}$;

(ii) system (1.1) has at least one solution for any $0<\lambda<\lambda^{*}$.

Furthermore, there exist a sequence $\left\{\lambda_{n}\right\}$ and a sequence solutions $\left\{\left(u_{\lambda_{n}}, v_{\lambda_{n}}\right)\right\}$ of system (1.1) satisfying $\lambda_{n} \rightarrow \lambda^{*}$ and $\left|u_{\lambda_{n}}\right|_{\infty}+\left|v_{\lambda_{n}}\right|_{\infty} \rightarrow \infty($ as $n \rightarrow \infty)$.

It is remarkable that under the assumption either $\delta=p-1$ and $0<\gamma<q-1$ or $\gamma=q-1$ and $0<\delta<p-1$ in system (1.1), when $\left(H_{2}\right)$ holds, we can also derive the same result as described in Theorem 3 .

The rest of this paper is organized as follows. In Section 2 we present some preliminary results which will be used in the proofs of our main results. In Section 3 we prove Theorem 1, and in Section 4 we prove Theorems 2 and 3, respectively. 


\section{Preliminaries}

In order to present proofs of our main results in a straightforward way, in this section we recall some basic lemmas, which include the maximum principle for the $p$-Laplacian operator [11]:

Lemma 1. Assume that $\Omega \subset R^{N}$ is a bounded domain of class $C^{1+\eta}, 0<\eta<1$, and suppose that $a \in L^{\infty}(\Omega)$. Then the following assertions are equivalent:

(i) $\mathscr{L}=-\Delta_{p}+a \varphi_{p}$ satisfies the maximum principle, where $\varphi_{p}(s)=|s|^{p-2} s$;

(ii) $\mathscr{L}=-\Delta_{p}+a \varphi_{p}$ satisfies the strong maximum principle;

(iii) $\lambda_{1, p}(a)>0$, where $\lambda_{1, p}(a)$ denotes the first eigenvalue of $\mathscr{L}$ with the homogeneous Dirichlet boundary condition;

(iv) there exists a positive and strict supersolution $\phi \in W^{1, p}(\Omega) \cap L^{\infty}(\Omega)$ of $\mathscr{L} u=0$ in $\Omega$ and $u=0$ on $\partial \Omega$, such that $-\Delta_{p} \phi+a(x) \varphi_{p}(\phi) \in L^{\infty}(\Omega)$ and $\left.\phi\right|_{\partial \Omega} \in C^{1+\eta}(\partial \Omega)$.

Suppose that $P$ is a cone of the real Banach space $X$ and ' $\leq$ ' denotes the order produced by $P$. We say that $P$ is normal if and only if each order interval in $X$ is bounded. Let $X^{\prime} \subset X$. An operator $\mathcal{N}$ is said to be an increasing operator in $X^{\prime}$ if any $x_{1}, x_{2} \in X^{\prime}$ satisfying $x_{1} \leq x_{2}$ implies $\mathcal{N} x_{1} \leq \mathcal{N} x_{2}$. [1].

The following is a lemma regarding a fixed point for the increasing operator

Lemma 2. Suppose that $x_{0}, y_{0} \in X$ with $x_{0} \leq y_{0}$, and $\mathcal{N}:\left[x_{0}, y_{0}\right] \rightarrow X$ is an increasing operator satisfying

$$
x_{0} \leq \mathcal{N} x_{0}, \quad \mathcal{N} y_{0} \leq y_{0} .
$$

If $P$ is normal and $\mathcal{N}$ is a completely continuous operator, then $\mathcal{N}$ has a minimal fixed point $x_{*}$ and a maximal fixed point $y^{*}$ in the interval $\left[x_{0}, y_{0}\right]$ such that

$$
x_{*}=\lim _{n \rightarrow \infty} x_{n}, \quad y^{*}=\lim _{n \rightarrow \infty} y_{n}
$$

where $x_{n}=\mathcal{N} x_{n-1}$ and $y_{n}=\mathcal{N} y_{n-1}$ satisfy

$$
x_{0} \leq x_{1} \leq \ldots \leq x_{n} \leq \ldots \leq x_{*} \leq y^{*} \leq \ldots \leq y_{n} \leq \ldots \leq y_{1} \leq y_{0} .
$$

Consider a parameter-dependent problem of the form

$$
x-F(\lambda, x)=0
$$

in a Banach space $X$, where $x \in X$ and $\lambda \in \mathbb{R}$. Solutions of equation (2.5) can be described by the following global continuation theorem, which is actually a global version of the implicit function theorem $[\mathbf{3}, \mathbf{8}]$.

Lemma 3. Let $F: \mathbb{R} \times X \rightarrow X$ be completely continuous and $\left(\lambda_{0}, x_{0}\right)$ be a solution of equation (2.5). Suppose that $\mathcal{U}$ is a bounded and open set such that $x_{0} \in \mathcal{U}$ and

(i) for fixed $\lambda_{0}$ there is no other solution in $\overline{\mathcal{U}}$;

(ii) the Leray-Schauder degree $\operatorname{deg}\left(I-F\left(\lambda_{0}, \cdot\right), \mathcal{U}, 0\right) \neq 0$.

Then there exists a closed and connected (=continuum) $S^{+} \subset\left[\lambda_{0}, \infty\right) \times X$ of solutions of system (2.5) with $\left(\lambda_{0}, x_{0}\right) \in S^{+}$and one of the following two alternative holds:

(a) $S^{+}$is unbounded,

(b) $S^{+} \cap\left(\left\{\lambda_{0}\right\} \times X \backslash \overline{\mathcal{U}}\right) \neq \emptyset$. 
To find a priori estimates for solutions of system (1.1), we need the lemma [16]:

LEMmA 4. Let $1<p, q<N$ and $\alpha \beta>(p-1)(q-1)$. Suppose that

$$
\max \left\{N_{1}-\frac{N-p}{p-1}, \quad N_{2}-\frac{N-q}{q-1}\right\} \geq 0 .
$$

Then the system

$$
\begin{cases}-\Delta_{p} u \geq c_{1} v^{\alpha}, & x \in \mathbb{R}^{N}, \\ -\Delta_{q} v \geq c_{2} u^{\beta}, & x \in \mathbb{R}^{N}, \\ u, v>0, & \end{cases}
$$

has no solution $(u, v) \in C^{1}\left(\mathbb{R}^{N}\right) \times C^{1}\left(\mathbb{R}^{N}\right)$ satisfying $|u|_{\infty} \leq 1$ and $|v|_{\infty} \leq 1$, where $c_{1}$ and $c_{2}$ are two positive constants.

\section{Proof of Theorem 1}

In this section, our objective is to investigate the existence and nonexistence of solutions of system (1.1) with $\Omega=B_{R}$. It follows from the moving plane method that any solutions of system (1.1) are all radially symmetric (see [15]). Denote

$$
\mathfrak{L}(I, \mathbb{R})=\{u \in C(I, \mathbb{R}): u(R)=0\}, \quad D=\mathfrak{L}(I, \mathbb{R}) \times \mathfrak{L}(I, \mathbb{R}),
$$

where $I=[0, R]$. Apparently, $D$ is a real Banach space induced by the maximum norm and

$$
P=\{(u, v) \in D:(u, v) \geq(0,0)\}
$$

is a normal cone in $D$. In order to show the branch of solutions of system (1.1), we need to define the following solution-operator $\mathcal{N}$ associated with system (1.1) by

$$
\mathcal{N}(\lambda, u, v)=(\bar{u}, \bar{v}),
$$

where $h_{t}(s)=|s|^{t} \operatorname{sign}(s)$, and

$$
\begin{aligned}
& \bar{u}(r)=\int_{r}^{R} h_{1 /(p-1)}\left(s^{1-N} \int_{0}^{s} t^{N-1} \lambda\left(a+|u(t)|^{\delta}+|v(t)|^{\alpha}\right) d t\right) d s \\
& \bar{v}(r)=\int_{r}^{R} h_{1 /(q-1)}\left(s^{1-N} \int_{0}^{s} t^{N-1} \lambda\left(b+|u(t)|^{\beta}+|v(t)|^{\gamma}\right) d t\right) d s
\end{aligned}
$$

We denote the norm of the Banach space $D$ by

$$
|(u, v)|_{\infty}=|u|_{\infty}+|v|_{\infty}
$$

By the Arzela-Ascoli Theorem, we know that $\mathcal{N}:[0, \infty) \times D \rightarrow D$ is completely continuous. It is easy to see that $(u, v) \in D$ solves system $(1.1)$, namely, $(u(|x|), v(|x|))$ is a solution of system (1.1) if and only if $(u, v)$ is a fixed point of $\mathcal{N}(\lambda, \cdot, \cdot)$.

Proposition 1. Assume that $\delta \geq p-1$ or $\gamma \geq q-1$. Then system (1.1) has no solution for sufficiently large $\lambda>0$.

Proof of Proposition 1. The assumption $\delta \geq p-1$ implies $\lambda\left(a+s^{\delta}+t^{\alpha}\right)>$ $\lambda\left(a+s^{\delta}\right)>\lambda_{1, p}(0) s^{p-1}$ for any $s, t>0$ and large $\lambda>0$, where $\lambda_{1, p}(0)$ denotes the first eigenvalue of $-\Delta_{p}$ with the homogeneous Dirichlet boundary condition. Suppose that $(u, v)$ is a solution of system (1.1), then we have

$$
\begin{cases}-\Delta_{p} u-\lambda_{1, p}(0) u^{p-1}>0, & x \in \Omega, \\ u=0, & x \in \partial \Omega .\end{cases}
$$


By the regularity argument, we have $(u, v) \in C_{0}^{1+\eta}(\bar{\Omega}) \times C_{0}^{1+\eta}(\bar{\Omega})$, where $0<\eta<1$. It follows from Lemma 1 that $\lambda_{1, p}\left(-\lambda_{1, p}(0)\right)>0$. This yields a contradiction to $\lambda_{1, p}\left(-\lambda_{1, p}(0)\right)=0$. For the case of $\gamma \geq q-1$, the proof is closely similar, so we omit it.

Since $(0,0)$ is only one fixed point of $\mathcal{N}$ at $\lambda=0$ and $\mathcal{N}(0, \cdot, \cdot)=0$, by the normal property of the Leray-Schauder degree, it follows that

Proposition 2. For a bounded and open subset $\mathcal{U} \subset D$ and $(0,0) \in \mathcal{U}$, we have

$$
\operatorname{deg}(I-\mathcal{N}(0, \cdot, \cdot), \mathcal{U}, 0)=1
$$

Now, we give a priori estimates for solutions of system (1.1) by using the scaling argument, which plays a critical role in the proof of Theorem 1.

Proposition 3. Let $1<p$ and $q<N$. Suppose that

$$
\begin{gathered}
\alpha \beta>(p-1)(q-1), \\
\max \left\{N_{1}-\frac{N-p}{p-1}, N_{2}-\frac{N-q}{q-1}\right\} \geq 0,
\end{gathered}
$$

and

$$
0<\delta<(p-1)+p / N_{1}, \quad 0<\gamma<(q-1)+q / N_{2} .
$$

Then for any $\bar{\lambda}>\underline{\lambda}>0$, there exists $M>0$ such that

$$
\left|u_{\lambda}\right|_{\infty}+\left|v_{\lambda}\right|_{\infty} \leq M
$$

holds for $\lambda \in[\underline{\lambda}, \bar{\lambda}]$ and any fixed point $\left(u_{\lambda}, v_{\lambda}\right) \in D$ of $\mathcal{N}(\lambda, \cdot, \cdot)$.

Proof of Proposition 3. Suppose that there exists a sequence $\lambda_{n} \in[\underline{\lambda}, \bar{\lambda}]$ such that the corresponding $\mathcal{N}\left(\lambda_{n}, \cdot, \cdot\right)$ has a fixed point $\left(u_{n}, v_{n}\right) \in D$ satisfying

$$
\left|u_{n}\right|_{\infty}+\left|v_{n}\right|_{\infty} \rightarrow \infty \quad(n \rightarrow \infty) .
$$

From (3.6) and (3.7), it follows that $u_{n}$ and $v_{n}$ attain the maximum values at 0 and $u_{n}^{\prime}(r), v_{n}^{\prime}(r) \leq 0$. Let $(\sigma, l)$ be the solution of the following linear system:

$$
\left\{\begin{array}{l}
\sigma(1-p)-p+\alpha l=0 \\
l(1-q)-q+\beta \sigma=0
\end{array}\right.
$$

that is,

$$
l=\frac{p q-q+\beta p}{\alpha \beta-(p-1)(q-1)}>0, \quad \sigma=\frac{p q-p+\alpha q}{\alpha \beta-(p-1)(q-1)}>0 .
$$

Denote

$$
u_{n}(0)=\mu_{n}, \quad v_{n}(0)=\tau_{n}, \quad C_{n}=\mu_{n}^{1 / \sigma}+\tau_{n}^{1 / l} .
$$

It is clear that $C_{n} \rightarrow \infty$ as $n \rightarrow \infty$. Assume $\mu_{n}^{1 / \sigma} \geq \tau_{n}^{1 / l}$ and denote

$$
\tilde{u}_{n}(\tilde{r})=\frac{u_{n}\left(C_{n}^{-1} \tilde{r}\right)}{A_{n}}, \quad \tilde{v}_{n}(\tilde{r})=\frac{v_{n}\left(C_{n}^{-1} \tilde{r}\right)}{B_{n}},
$$


where $A_{n}=C_{n}^{\sigma}$ and $B_{n}=C_{n}^{l}$. Thus, we have

$$
\begin{aligned}
& -\left[\tilde{r}^{N-1} h_{p-1}\left(\tilde{u}_{n}^{\prime}(\tilde{r})\right)\right]^{\prime} \\
= & -\left[\tilde{r}^{N-1} h_{p-1}\left(\frac{u_{n}^{\prime}\left(\tilde{r} C_{n}^{-1}\right)}{A_{n} C_{n}}\right)\right]^{\prime} \\
= & -A_{n}^{1-p} C_{n}^{1-p}\left[\tilde{r}^{N-1} h_{p-1}\left(u_{n}^{\prime}\left(\tilde{r} C_{n}^{-1}\right)\right)\right]^{\prime} \\
= & -A_{n}^{1-p} C_{n}^{N-p-1}\left[r^{N-1} h_{p-1}\left(u_{n}^{\prime}(r)\right)\right]^{\prime} \\
= & r^{N-1} C_{n}^{N-p-1} A_{n}^{1-p}\left[\lambda_{n}\left(a+\left|u_{n}(r)\right|^{\delta}+\left|v_{n}(r)\right|^{\alpha}\right)\right] \\
= & \tilde{r}^{N-1}\left(a \lambda_{n} C_{n}^{-p} A_{n}^{1-p}+\lambda_{n} C_{n}^{-p} A_{n}^{\delta-p+1} \tilde{u}^{\delta}+\lambda_{n} C_{n}^{-p} A_{n}^{1-p} B_{n}^{\alpha} \tilde{v}_{n}^{\alpha}\right) .
\end{aligned}
$$

Similarly, we have

$-\left[\tilde{r}^{N-1} h_{q-1}\left(\tilde{v}_{n}^{\prime}(\tilde{r})\right)\right]^{\prime}=\tilde{r}^{N-1}\left(b \lambda_{n} C_{n}^{-q} B_{n}^{1-q}+\lambda_{n} C_{n}^{-q} B_{n}^{\gamma-q+1} \tilde{v}_{n}^{\gamma}+\lambda_{n} B_{n}^{1-q} C_{n}^{-q} A_{n}^{\beta} \tilde{u}_{n}^{\beta}\right)$.

Without loss of generality, we assume $\lambda_{n} \rightarrow \lambda_{0} \in[\underline{\lambda}, \bar{\lambda}]$ as $n \rightarrow \infty$. It is clear that

Since

$$
\begin{aligned}
& a \lambda_{n} C_{n}^{-p} A_{n}^{1-p} \rightarrow 0 \quad(n \rightarrow \infty), \\
& b \lambda_{n} C_{n}^{-q} B_{n}^{1-q} \rightarrow 0 \quad(n \rightarrow \infty) .
\end{aligned}
$$

we have

$$
0<\delta<(p-1)+p / N_{1}, \quad 0<\gamma<(q-1)+q / N_{2},
$$

and

$$
\sigma \delta-p+(1-p) \sigma<0, \quad l(1-q)-q+l \gamma<0,
$$

$$
\begin{aligned}
& \lambda_{n} C_{n}^{-p} A_{n}^{\delta-p+1} \rightarrow 0 \quad(n \rightarrow \infty), \\
& \lambda_{n} C_{n}^{-q} B_{n}^{\gamma-q+1} \rightarrow 0 \quad(n \rightarrow \infty) .
\end{aligned}
$$

For the sake of conciseness, we denote

$$
w_{n}(x)=\tilde{u}_{n}(|x|), \quad z_{n}(x)=\tilde{v}_{n}(|x|)
$$

for each $x \in B_{C_{n} R}(0)$, then we have that

$$
\text { (3.9) }\left\{\begin{array}{l}
-\Delta_{p} w_{n}=a \lambda_{n} C_{n}^{-p} A_{n}^{1-p}+\lambda_{n} C_{n}^{-p} A_{n}^{\delta-p+1} w_{n}^{\delta}+\lambda_{n} C_{n}^{-p} A_{n}^{1-p} B_{n}^{\alpha} z_{n}^{\alpha}, \\
-\Delta_{q} z_{n}=b \lambda_{n} C_{n}^{-q} B_{n}^{1-q}+\lambda_{n} C_{n}^{-q} B_{n}^{\gamma-q+1} z_{n}^{\gamma}+\lambda_{n} C_{n}^{-q} B_{n}^{1-q} A_{n}^{\beta} w_{n}^{\beta} .
\end{array}\right.
$$

Since $0 \leq w_{n}$ and $z_{n} \leq 1$, by regularity arguments for quasilinear elliptic equations $[\mathbf{1 8}]$, it follows that $\left\{\left(w_{n}, z_{n}\right)\right\}$ has a convergent subsequence in $C_{l o c}^{1}\left(\mathbb{R}^{N}\right) \times$ $C_{l o c}^{1}\left(\mathbb{R}^{N}\right)$. Without loss of generality, we assume that

$$
w_{n} \rightarrow w, \quad z_{n} \rightarrow z \quad(\text { as } n \rightarrow \infty)
$$

in $C_{l o c}^{1}\left(\mathbb{R}^{N}\right)$. Since

we have

$$
\tilde{u}_{n}(0)=\mu_{n} /\left(\mu_{n}^{1 / \sigma}+\tau_{n}^{1 / l}\right)^{\sigma}
$$

$$
\frac{1}{2^{\sigma}} \leq w(0) \leq 1
$$

Using the maximum principle, we can deduce that

$$
\begin{cases}-\Delta_{p} w=\lambda_{0} z^{\alpha}, & x \in \mathbb{R}^{N}, \\ -\Delta_{q} z=\lambda_{0} w^{\beta}, & x \in \mathbb{R}^{N},\end{cases}
$$

where $|w|_{\infty} \leq 1,|z|_{\infty} \leq 1, w>0$ and $z>0$, which gives a contradiction with Lemma 4.

When $\tau_{n}^{1 / l} \geq \mu_{n}^{1 / \sigma}$, the argument is almost the same. 
Remark 1. Proposition 3 gives a priori bounds of maximum norm for solutions. Indeed, for any $\bar{\lambda}>\underline{\lambda}>0$, by combining with regularity arguments for quasilinear elliptic equations, there exists $M^{\prime}>0$ such that

$$
\left|\left(u_{\lambda}, v_{\lambda}\right)\right|_{\infty}+\left|\left(u_{\lambda}^{\prime}, v_{\lambda}^{\prime}\right)\right|_{\infty} \leq M^{\prime}
$$

for each $\lambda \in[\underline{\lambda}, \bar{\lambda}]$ and any fixed point $\left(u_{\lambda}, v_{\lambda}\right) \in D$ of $\mathcal{N}(\lambda, \cdot, \cdot)$.

Proof of Theorem 1 . When $\lambda=0, \mathcal{N}(0, \cdot, \cdot)$ has a unique fixed point $(0,0)$. By Lemma 3 and Proposition 2, there exists an unbounded continuum $S^{+} \subset$ $[0, \infty) \times D$ of solutions of $(u, v)=\mathcal{N}(\lambda, u, v)$. It follows from Proposition 1 that the continuum $S^{+}$is bounded in the $\lambda$-direction, so it has to become unbounded in the direction of the Banach space $D$. In virtue of Proposition $3, S^{+}$bends back to $\lambda=0$ and becomes unbounded near $\lambda=0$. Let $S \subset[0, \infty) \times D$ be the set containing all solutions of $(u, v)=\mathcal{N}(\lambda, u, v)$. In order to make $S^{+}$maximally connected we replace $S^{+}$by the connected components of $S$ containing $S^{+}$. For our convenience, we still denote it by $S^{+}$. Let $\left[0, \lambda^{*}\right]$ be the projection of $S^{+}$onto the $\lambda$-direction. We still denote $u(|x|)$ by $u(x)$, and the other radially symmetric functions are also denoted in this way.

Step 1 For any $\lambda>\lambda^{*}$, system (1.1) has no radially symmetric solution. Otherwise, there exists $\mu>\lambda^{*}$ such that system (1.1) with $\lambda=\mu$ has a radially symmetric solution, and we denote it by $\left(u_{\mu}, v_{\mu}\right)$. Since $\mathcal{N}(\mu, 0,0) \geq(0,0)$ and $\mathcal{N}\left(\mu, u_{\mu}, v_{\mu}\right)=\left(u_{\mu}, v_{\mu}\right)$, in virtue of Lemma $2, \mathcal{N}(\mu, \cdot, \cdot)$ has a minimal fixed point $\left(\underline{u}_{\mu}, \underline{v}_{\mu}\right)$. Denote

$$
\begin{gathered}
V=[0, \mu] \times\left\{(u, v) \in D_{1}: 0<u(x)<\underline{u}_{\mu}(x), \quad 0<v(x)<\underline{v}_{\mu}(x), \quad \forall x \in \Omega,\right. \\
\text { and } \left.0>\frac{\partial u(x)}{\partial \nu}>\frac{\partial \underline{u}_{\mu}(x)}{\partial \nu}, \quad 0>\frac{\partial v(x)}{\partial \nu}>\frac{\partial \underline{v}_{\mu}(x)}{\partial \nu}, \quad \forall x \in \partial \Omega\right\},
\end{gathered}
$$

where $D_{1}$ consists of all radially symmetric functions in $C_{0}^{1}(\bar{\Omega}) \times C_{0}^{1}(\bar{\Omega})$. Since $(0,0,0) \in S^{+}$, for sufficiently small $\lambda>0$, there exists the corresponding $\left(\lambda, u_{\lambda}, v_{\lambda}\right) \in$ $S^{+}$such that

$$
\lambda \max \left\{\left(a+u_{\lambda}^{\delta}+v_{\lambda}^{\alpha}\right),\left(b+u_{\lambda}^{\beta}+v_{\lambda}^{\gamma}\right)\right\}<\mu \min \{a, b\} .
$$

So, the strong comparison principle implies $S^{+} \cap V \neq \emptyset$. If there exist $\lambda \in(0, \mu)$ and the corresponding solution $\left(\lambda, u_{\lambda}, v_{\lambda}\right) \in S^{+}$satisfying $\left(\lambda, u_{\lambda}, v_{\lambda}\right) \in \bar{V}$, then by the strong comparison principle [6], we have $\left(\lambda, u_{\lambda}, v_{\lambda}\right) \in V$. Hence, $S^{+}$stays inside $V$, which is a contradiction to the unboundedness of $S^{+}$.

Step 2 For any $\lambda \in\left(0, \lambda^{*}\right)$, it holds that $\left(\lambda, \underline{u}_{\lambda}, \underline{v}_{\lambda}\right) \in S^{+}$, where $\left(\underline{u}_{\lambda}, \underline{v}_{\lambda}\right)$ is a minimal fixed point of $\mathcal{N}(\lambda, \cdot, \cdot)$. Suppose that there exists $\lambda^{\prime} \in\left(0, \lambda^{*}\right)$ such that $\left(\lambda^{\prime}, \underline{u}_{\lambda^{\prime}}, \underline{v}_{\lambda^{\prime}}\right) \notin S^{+}$, where $\left(\underline{u}_{\lambda^{\prime}}, \underline{v}_{\lambda^{\prime}}\right)$ is a minimal fixed point of $\mathcal{N}\left(\lambda^{\prime}, \cdot, \cdot\right)$. Denote

$$
\begin{gathered}
V=\left[0, \lambda^{\prime}\right] \times\left\{(u, v) \in D_{1}: 0<u(x)<\underline{u}_{\lambda^{\prime}}(x), \quad 0<v(x)<\underline{v}_{\lambda^{\prime}}(x), \quad \forall x \in \Omega,\right. \\
\text { and } \left.0>\frac{\partial u(x)}{\partial \nu}>\frac{\partial \underline{u}_{\lambda^{\prime}}(x)}{\partial \nu}, \quad 0>\frac{\partial v(x)}{\partial \nu}>\frac{\partial \underline{v}_{\lambda^{\prime}}(x)}{\partial \nu}, \quad \forall x \in \partial \Omega\right\} .
\end{gathered}
$$

Using a similar argument as that given in Step 1, we can obtain a contradiction to the unboundedness of $S^{+}$too.

Step 3 For any $\lambda \in\left(0, \lambda^{*}\right)$, there exists $\left(\lambda, \bar{u}_{\lambda}, \bar{v}_{\lambda}\right) \in S^{+}$such that

$$
\left(\bar{u}_{\lambda}, \bar{v}_{\lambda}\right) \not \leq\left(\underline{u}_{\lambda^{*}}, \underline{v}_{\lambda^{*}}\right),
$$


where $\left(\underline{u}_{\lambda^{*}}, \underline{v}_{\lambda^{*}}\right)$ is a minimal fixed point of $\mathcal{N}\left(\lambda^{*}, \cdot, \cdot\right)$. Otherwise, there exists $\tilde{\lambda} \in\left(0, \lambda^{*}\right)$ such that for any $(\tilde{\lambda}, \tilde{u}, \tilde{v}) \in S^{+}$we have

$$
(\tilde{u}, \tilde{v}) \leq\left(\underline{u}_{\lambda^{*}}, \underline{v}_{\lambda^{*}}\right) \text {. }
$$

Let

$U=[0, \tilde{\lambda}) \times\left\{(u, v) \in D_{1}: \exists x \in \Omega\right.$ such that $u(x)>\underline{u}_{\lambda^{*}}(x)$ or $\left.v(x)>\underline{v}_{\lambda^{*}}(x)\right\}$.

$U$ is a relatively open set for $[0, \infty) \times D_{1}$. Since $S^{+}$becomes unbounded near $\lambda=0$, we have $S^{+} \cap U \neq \emptyset$. It is easy to see $S^{+} \cap \bar{U}^{c} \neq \emptyset$ and connectedness of $S^{+}$implies $S^{+} \cap \partial U \neq \emptyset$. So we have

$$
\partial U=(\{\tilde{\lambda}\} \times \bar{Z}) \cup([0, \tilde{\lambda}] \times \partial Z),
$$

where

$$
Z=\left\{(u, v) \in D_{1}: \exists x \in \Omega \text { such that } u(x)>\underline{u}_{\lambda^{*}}(x) \text { or } v(x)>\underline{v}_{\lambda^{*}}(x)\right\} .
$$

Let

$$
\begin{aligned}
\bar{Z}_{1}= & \left\{(u, v) \in D_{1}: \exists x \in \partial \Omega \text { such that } \frac{\partial u(x)}{\partial \nu} \leq \frac{\partial \underline{u}_{\lambda^{*}}(x)}{\partial \nu}\right. \\
& \text { or } \left.\frac{\partial v(x)}{\partial \nu} \leq \frac{\partial \underline{v}_{\lambda^{*}}(x)}{\partial \nu}\right\}, \\
\bar{Z}_{2}= & \left\{(u, v) \in D_{1}: \exists x \in \Omega \text { such that } u(x) \geq \underline{u}_{\lambda^{*}}(x) \text { or } v(x) \geq \underline{v}_{\lambda^{*}}(x)\right\} .
\end{aligned}
$$

So we see that $\bar{Z}=\bar{Z}_{1} \cup \bar{Z}_{2}$.

By (3.11) and the strong comparison principle, we have

$$
S^{+} \cap(\{\tilde{\lambda}\} \times \bar{Z})=\emptyset,
$$

which implies

$$
([0, \tilde{\lambda}] \times \partial Z) \cap S^{+} \neq \emptyset .
$$

Clearly, it holds that $\bar{Z}^{c}=Y_{1} \cap Y_{2}$, where

$$
\begin{aligned}
& Y_{1}=\left\{(u, v) \in D_{1}: u(x)<\underline{u}_{\lambda^{*}}(x), \quad v(x)<\underline{v}_{\lambda^{*}}(x), \quad \forall x \in \Omega\right\}, \\
& Y_{2}=\left\{(u, v) \in D_{1}: \frac{\partial u(x)}{\partial \nu}>\frac{\partial \underline{u}_{\lambda^{*}}(x)}{\partial \nu}, \frac{\partial v(x)}{\partial \nu}>\frac{\partial \underline{v}_{\lambda^{*}}(x)}{\partial \nu}, \forall x \in \partial \Omega\right\} .
\end{aligned}
$$

Hence, we see $\partial Z=\partial\left(\bar{Z}^{c}\right)$. Using the strong comparison principle again gives $([0, \tilde{\lambda}] \times \partial Z) \cap S^{+}=\emptyset$, which is a contradiction.

By Lemma 2 , for any $\lambda \in\left(0, \lambda^{*}\right)$, we have $\left(\underline{u}_{\lambda}, \underline{v}_{\lambda}\right) \leq\left(\underline{u}_{\lambda^{*}}, \underline{v}_{\lambda^{*}}\right)$. Consequently, for any $\lambda \in\left(0, \lambda^{*}\right)$, system (1.1) has at least two solutions. When $\lambda=\lambda^{*}$, by virtue of compact arguments, system (1.1) has a solution. Since solutions of system (1.1) are all radially symmetric, From the discussion in Step 1 it indicates that system (1.1) has no solution for any $\lambda>\lambda^{*}$.

REMARK 2. From the above proof, one can see that the condition (1.4) can actually be weakened. Theorem 1 still holds if (1.4) is replaced by the following condition:

$$
0<\delta<(p-1)+p / N_{1}, \quad 0<\gamma<(q-1)+q / N_{2},
$$

and

$$
\delta \geq p-1 \text { or } \gamma \geq q-1
$$


REMARK 3. We can also apply the same idea to consider a more general problem

$$
\begin{cases}-\Delta_{p} u=\lambda\left(a_{1}+f_{1}(u)+g_{1}(v)\right), & x \in \Omega, \\ -\Delta_{q} v=\lambda\left(a_{2}+f_{2}(u)+g_{2}(v)\right), & x \in \Omega, \\ u>0, \quad v>0, & x \in \Omega, \\ u=v=0, & x \in \partial \Omega,\end{cases}
$$

where $a_{1}$ and $a_{2}$ are positive constants. If $f_{1}, f_{2}, g_{1}$ and $g_{2}$ are nondecreasing and continuous functions, and there are $c_{1}, c_{2}>0$ such that for any $s>0$,

$$
\begin{aligned}
& c_{1} s^{\delta} \leq f_{1}(s) \leq c_{2} s^{\delta}, \quad c_{1} s^{\beta} \leq f_{2}(s) \leq c_{2} s^{\beta}, \\
& c_{1} s^{\alpha} \leq g_{1}(s) \leq c_{2} s^{\alpha}, \quad c_{1} s^{\gamma} \leq g_{2}(s) \leq c_{2} s^{\gamma},
\end{aligned}
$$

hold, then Theorem 1 is true for system (3.12).

\section{Proofs of Theorems 2 and 3}

In this section, we assume that $\alpha \beta<(p-1)(q-1)$ and $\Omega$ is a bounded and smooth domain. We denote the norm of $W_{0}^{1, p}(\Omega)$ and $W_{0}^{1, q}(\Omega)$ by $\|\cdot\|_{p}$ and $\|\cdot\|_{q}$ as follows, respectively

$$
\|u\|_{p}=\left(\int_{\Omega}|\nabla u|^{p} d x\right)^{\frac{1}{p}}, \quad\|v\|_{q}=\left(\int_{\Omega}|\nabla v|^{q} d x\right)^{\frac{1}{q}} .
$$

Let

$$
E_{0}=C_{0}(\bar{\Omega}) \times C_{0}(\bar{\Omega})
$$

where $C_{0}(\bar{\Omega})=\left\{u \in C(\bar{\Omega}):\left.u\right|_{\partial \Omega}=0\right\}$.

For any weak solution belonging to $E_{0}$, the regularity arguments imply that the weak solution belongs to $C_{0}^{1+\eta}(\bar{\Omega}) \times C_{0}^{1+\eta}(\bar{\Omega})$, where $0<\eta<1$. So, using a similar argument to the proof of Proposition 1 , we obtain

Proposition 4. Suppose that $\delta=p-1$ or $\gamma=q-1$. Then there exists $\lambda_{0}>0$ such that for any $\lambda>\lambda_{0}$ system (1.1) has no weak solution belonging to $E_{0}$.

Let

$$
\lambda_{0}=\sup \{\lambda>0: \text { syetem }(1.1) \text { has a solution }\} \text {. }
$$

REMARK 4. Suppose that $\delta=p-1$ and $\gamma=q-1$. According to Proposition 4 , if there exists some $\lambda>0$ such that the corresponding system (1.1) has a weak solution belonging to $E_{0}$, then $\lambda_{0}<\infty$. Moreover, it follows from Lemma 1 that $\lambda_{0} \leq \min \left\{\lambda_{1, p}(0), \lambda_{1, q}(0)\right\}$.

Proposition 5. Suppose that $\left(H_{1}\right)$ and $\left(H_{2}\right)$ hold. Then for any $\bar{\lambda}>0$, there exists $M>0$ such that

$$
\left\|u_{\lambda}\right\|_{p} \leq M, \quad\left\|v_{\lambda}\right\|_{q} \leq M
$$

hold for $\lambda \in[0, \bar{\lambda}]$ and each weak solution $\left(u_{\lambda}, v_{\lambda}\right)$ of system (1.1). 
Proof of Proposition 5. Suppose that $\lambda \in[0, \bar{\lambda}]$ and $\left(u_{\lambda}, v_{\lambda}\right)$ is an arbitrary weak solution of system (1.1). Then, we have

$$
\begin{aligned}
\int_{\Omega}\left|\nabla u_{\lambda}\right|^{p} & =\lambda \int_{\Omega}\left(a+u_{\lambda}^{\delta}+v_{\lambda}^{\alpha}\right) u_{\lambda} \leq \bar{\lambda} \int_{\Omega}\left(a+u_{\lambda}^{\delta}+v_{\lambda}^{\alpha}\right) u_{\lambda}, \\
\int_{\Omega}\left|\nabla v_{\lambda}\right|^{q} & =\lambda \int_{\Omega}\left(b+u_{\lambda}^{\beta}+v_{\lambda}^{\gamma}\right) v_{\lambda} \leq \bar{\lambda} \int_{\Omega}\left(b+u_{\lambda}^{\beta}+v_{\lambda}^{\gamma}\right) v_{\lambda} .
\end{aligned}
$$

Denote by $C(\bar{\lambda})$ and $c(\bar{\lambda})$ the constants only depending on $\bar{\lambda}$, $\Omega$ and the best Sobolev constants for the embedding, but they may vary from lines to lines. Due to (4.2), one can see

that is,

$$
\left\|u_{\lambda}\right\|_{p}^{p} \leq C(\bar{\lambda})+\frac{1}{2} \int_{\Omega}\left|\nabla u_{\lambda}\right|^{p}+\bar{\lambda} \int_{\Omega}\left(u_{\lambda}^{\delta+1}+u_{\lambda} v_{\lambda}^{\alpha}\right)
$$

$$
\left\|u_{\lambda}\right\|_{p}^{p} \leq C(\bar{\lambda})+2 \bar{\lambda} \int_{\Omega}\left(u_{\lambda}^{\delta+1}+u_{\lambda} v_{\lambda}^{\alpha}\right) .
$$

Since $\delta+1<p$, it follows Young's inequality that

$$
\left\|u_{\lambda}\right\|_{p}^{p} \leq C(\bar{\lambda})+c(\bar{\lambda}) \int_{\Omega} u_{\lambda} v_{\lambda}^{\alpha} .
$$

By $\frac{p}{p^{*}}+\frac{\alpha}{q^{*}}<1$ (implies $\frac{1}{p^{*}}+\frac{\alpha}{q^{*}}<1$ ) and Hölder's inequality, inequality (4.4) implies that

$$
\begin{aligned}
\left\|u_{\lambda}\right\|_{p}^{p} & \leq C(\bar{\lambda})+c(\bar{\lambda})\left(\int_{\Omega}\left|u_{\lambda}\right|^{p^{*}}\right)^{\frac{1}{p^{*}}}\left(\int_{\Omega}\left|v_{\lambda}\right|^{q^{*}}\right)^{\frac{\alpha}{q^{*}}} \\
& \leq C(\bar{\lambda})+c(\bar{\lambda})\left\|u_{\lambda}\right\|_{p}\left\|v_{\lambda}\right\|_{q}^{\alpha} .
\end{aligned}
$$

Similarly, we have

$$
\left\|v_{\lambda}\right\|_{q}^{q} \leq C(\bar{\lambda})+c(\bar{\lambda})\left\|u_{\lambda}\right\|_{p}^{\beta}\left\|v_{\lambda}\right\|_{q} .
$$

Suppose that (4.1) is not true, then there exist a sequence $\lambda_{n} \in[0, \bar{\lambda}]$ and the corresponding $\left(u_{\lambda_{n}}, v_{\lambda_{n}}\right)$ such that

$$
\left\|u_{\lambda_{n}}\right\|_{p} \rightarrow \infty \text { or }\left\|v_{\lambda_{n}}\right\|_{q} \rightarrow \infty \text {. }
$$

For our convenience, let $u_{n}=u_{\lambda_{n}}$ and $v_{n}=v_{\lambda_{n}}$. If $\left\|u_{n}\right\|_{p} \rightarrow \infty$, by (4.5) it implies $\left\|v_{n}\right\|_{q} \rightarrow \infty$. If $\left\|v_{n}\right\|_{q} \rightarrow \infty$, by (4.6) it implies $\left\|u_{n}\right\|_{p} \rightarrow \infty$. So, we have

$$
\left\|u_{n}\right\|_{p} \rightarrow \infty \text { and }\left\|v_{n}\right\|_{q} \rightarrow \infty
$$

From (4.5)-(4.6), it follows that

$$
\begin{aligned}
& 1 \leq o(1)+c(\bar{\lambda}) \frac{\left\|v_{n}\right\|_{q}^{\alpha}}{\left\|u_{n}\right\|_{p}^{p-1}}, \\
& 1 \leq o(1)+c(\bar{\lambda}) \frac{\left\|u_{n}\right\|_{p}^{\beta}}{\left\|v_{n}\right\|_{q}^{q-1}} .
\end{aligned}
$$

In virtue of (4.8) and (4.9) and letting $n$ be large enough, we have

$$
\left\|v_{n}\right\|_{q}^{\alpha} \geq \frac{1}{2 c(\bar{\lambda})}\left\|u_{n}\right\|_{p}^{p-1}, \quad\left\|u_{n}\right\|_{p}^{\beta} \geq \frac{1}{2 c(\bar{\lambda})}\left\|v_{n}\right\|_{q}^{q-1}
$$

It follows from (4.10) that

$$
\left\|v_{n}\right\|_{q}^{\alpha} \geq\left(\frac{1}{2 c(\bar{\lambda})}\right)^{(p-1+\beta) / \beta}\left\|v_{n}\right\|_{q}^{(q-1)(p-1) / \beta} .
$$


Since $(p-1)(q-1)>\alpha \beta$, it becomes impossible for (4.11) as $\left\|v_{n}\right\|_{q} \rightarrow \infty$. Consequently, the proof is completed.

Proposition 6. If $\delta=p-1, \gamma=q-1$ and $\left(H_{2}\right)$ holds, then for any given $0<\bar{\lambda}<\lambda_{0}$, there exists $M>0$ such that

$$
\left\|u_{\lambda}\right\|_{p} \leq M, \quad\left\|v_{\lambda}\right\|_{q} \leq M
$$

hold for $\lambda \in[0, \bar{\lambda}]$ and each weak solution $\left(u_{\lambda}, v_{\lambda}\right)$ of system (1.1).

Proof of Proposition 6. By $\left(H_{2}\right)$ and the regularity arguments, any weak solution of system (1.1) belongs to $E_{0}$. From Remark 4, it follows that $\lambda_{0} \leq$ $\min \left\{\lambda_{1, p}(0), \lambda_{1, q}(0)\right\}$. So we have

$$
\begin{aligned}
\int_{\Omega}\left|\nabla u_{\lambda}\right|^{p} & =\lambda \int_{\Omega}\left(a+u_{\lambda}^{\delta}+v_{\lambda}^{\alpha}\right) u_{\lambda} \leq \bar{\lambda} \int_{\Omega}\left(a+u_{\lambda}^{p-1}+v_{\lambda}^{\alpha}\right) u_{\lambda}, \\
\int_{\Omega}\left|\nabla v_{\lambda}\right|^{q} & =\lambda \int_{\Omega}\left(b+u_{\lambda}^{\beta}+v_{\lambda}^{\gamma}\right) v_{\lambda} \leq \bar{\lambda} \int_{\Omega}\left(b+u_{\lambda}^{q-1}+v_{\lambda}^{\gamma}\right) v_{\lambda} .
\end{aligned}
$$

By (4.12) and (4.13), we get

$$
\begin{aligned}
& \left(1-\frac{\bar{\lambda}}{\lambda_{1, p}(0)}-\epsilon\right) \int_{\Omega}\left|\nabla u_{\lambda}\right|^{p} \leq C(\bar{\lambda})+\bar{\lambda} \int_{\Omega} u_{\lambda} v_{\lambda}^{\alpha}, \\
& \left(1-\frac{\bar{\lambda}}{\lambda_{1, q}(0)}-\epsilon\right) \int_{\Omega}\left|\nabla v_{\lambda}\right|^{p} \leq C(\bar{\lambda})+\bar{\lambda} \int_{\Omega} v_{\lambda} u_{\lambda}^{\beta},
\end{aligned}
$$

where

$$
0<\epsilon<\min \left\{1-\frac{\bar{\lambda}}{\lambda_{1, p}(0)}, 1-\frac{\bar{\lambda}}{\lambda_{1, q}(0)}\right\} .
$$

By (4.14) and (4.15), a straightforward calculation leads to (4.5) and (4.6). The rest proof is similar to that of Proposition 5, so we omit it.

The following proposition is a regularity result, which also gives a priori estimates of the solution of system (1.1).

Proposition 7. Suppose that $\delta=p-1, \gamma=q-1$, and $\left(H_{2}\right)$ holds. Then for any given $\bar{\lambda}<\lambda_{0}$, there exists $M$ such that

$$
\left|u_{\lambda}\right|_{\infty} \leq M, \quad\left|v_{\lambda}\right|_{\infty} \leq M
$$

hold for $\lambda \in[0, \bar{\lambda}]$ and any weak solution $\left(u_{\lambda}, v_{\lambda}\right) \in W_{0}^{1, p}(\Omega) \times W_{0}^{1, q}(\Omega)$ of system (1.1).

Proof of Proposition 7. We will use the standard method of regularity arguments. Due to $\left(H_{2}\right)$, it follows that

$$
\mu=\frac{\left(q^{*}-\alpha\right) p^{*}}{p q^{*}}>1, \quad \frac{p \mu}{p^{*}}+\frac{\alpha}{q^{*}}=1 .
$$

Let

and

$$
Z_{L}=u_{\lambda} u_{L}^{p(\mu-1)}, \quad W_{L}=u_{\lambda} u_{L}^{\mu-1},
$$

$$
u_{L}= \begin{cases}L, & u_{\lambda}>L, \\ u_{\lambda}, & 0 \leq u_{\lambda} \leq L .\end{cases}
$$


Since

$$
\begin{aligned}
& \int_{\Omega}\left|Z_{L}\right|^{p} \leq \int_{\Omega} u_{\lambda}^{p} u_{L}^{p^{2}(\mu-1)}<+\infty \\
& \int_{\Omega}\left|D Z_{L}\right|^{p} \leq c \int_{\Omega}\left|D u_{\lambda}\right|^{p}+C \int_{\Omega}\left|u_{\lambda}\right|^{p} u_{L}^{p^{2}(\mu-1)-p}\left|D u_{L}\right|^{p} \\
& \leq C_{1}+C \int_{\left|u_{\lambda}\right| \leq L} u_{\lambda}^{p}\left|D u_{L}\right|^{p} \\
& \leq C_{1}+C \int_{\Omega}\left|D u_{\lambda}\right|^{p} \\
&<+\infty
\end{aligned}
$$

by the first equation of (1.1) we have

$$
\begin{aligned}
\int_{\Omega}\left|D u_{\lambda}\right|^{p} u_{L}^{p(\mu-1)}= & -p(\mu-1) \int_{\Omega} u_{\lambda} u_{L}^{p(\mu-1)-1}\left|D u_{\lambda}\right|^{p-2} D u_{\lambda} D u_{L} \\
& +\lambda a \int_{\Omega} Z_{L}+\lambda \int_{\Omega} u_{\lambda}^{p} u_{L}^{p(\mu-1)}+\lambda \int_{\Omega} v_{\lambda}^{\alpha} u_{\lambda} u_{L}^{p(\mu-1)}
\end{aligned}
$$

From (4.16), it follows that

$$
(4.17) \int_{\Omega}\left|D u_{\lambda}\right|^{p} u_{L}^{p(\mu-1)} \leq \bar{\lambda} a \int_{\Omega} Z_{L}+\bar{\lambda} \int_{\Omega} u_{\lambda}^{p} u_{L}^{p(\mu-1)}+\bar{\lambda} \int_{\Omega} u_{\lambda} u_{L}^{p(\mu-1)} v_{\lambda}^{\alpha} .
$$

Since

$$
\begin{aligned}
\int_{\Omega}\left|D W_{L}\right|^{p} & =\int_{\Omega}\left|D\left(u_{\lambda} u_{L}^{\mu-1}\right)\right|^{p} \\
& =\int_{\Omega}\left|D u_{\lambda} u_{L}^{\mu-1}+(\mu-1) u_{\lambda} u_{L}^{\mu-2} D u_{L}\right|^{p} \\
& \leq C \int_{\Omega}\left|D u_{\lambda}\right|^{p} u_{L}^{p(\mu-1)}+C(\mu-1)^{p} \int_{\Omega} u_{L}^{p(\mu-1)}\left|D u_{\lambda}\right|^{p} \\
& \leq C \mu^{p} \int_{\Omega}\left|D u_{\lambda}\right|^{p} u_{L}^{p(\mu-1)}
\end{aligned}
$$

by the Sobolev inequality and (4.17), we have

$$
\begin{aligned}
\left(\int_{\Omega}\left|W_{L}\right|^{p^{*}}\right)^{\frac{p}{p^{*}}} & \leq C \int_{\Omega}\left|D W_{L}\right|^{p} \leq C \mu^{p} \int_{\Omega}\left|D u_{\lambda}\right|^{p} u_{L}^{p(\mu-1)} \\
& \leq C \bar{\lambda} \mu^{p} \int_{\Omega}\left(Z_{L}+u_{\lambda}^{p} u_{L}^{p(\mu-1)}+u_{\lambda} u_{L}^{p(\mu-1)} v_{\lambda}^{\alpha}\right) \\
& =C \mu^{p} \int_{\Omega}\left(W_{L} u_{L}^{(p-1)(\mu-1)}+W_{L}^{p}+W_{L} u_{L}^{(p-1)(\mu-1)} v_{\lambda}^{\alpha}\right) \\
& \leq C \mu^{p} \int_{\Omega}\left(u_{\lambda}^{\mu+(p-1)(\mu-1)}+u_{\lambda}^{\mu p}+u_{\lambda}^{\mu+(p-1)(\mu-1)} v_{\lambda}^{\alpha}\right) .
\end{aligned}
$$

Using $\frac{\mu p}{p^{*}}+\frac{\alpha}{q^{*}}=1$ gives

$$
\frac{\alpha}{q^{*}}+\frac{\mu+(p-1)(\mu-1)}{p^{*}}<1 .
$$


In virtue of Hölder's inequality and Proposition 6, from (4.18) we have

$$
\begin{aligned}
& \left(\int_{\Omega}\left|W_{L}\right|^{p^{*}}\right)^{\frac{p}{p^{*}}} \leq \\
& C \mu^{p}\left\{\left(\int_{\Omega} u_{\lambda}^{p^{*}}\right)^{\frac{\mu+(p-1)(\mu-1)}{p^{*}}}+\left(\int_{\Omega} u_{\lambda}^{p^{*}}\right)^{\frac{\mu p}{p^{*}}}+\left(\int_{\Omega} u_{\lambda}^{p^{*}}\right)^{\frac{\mu+(p-1)(\mu-1)}{p^{*}}}\right\} .
\end{aligned}
$$

By making use of $\mu p>\mu+(p-1)(\mu-1)$, inequality (4.19) becomes

$$
\left(\int_{\Omega}\left|W_{L}\right|^{p^{*}}\right)^{\frac{p}{p^{*}}} \leq C \mu^{p} \max \left\{1,\left(\int_{\Omega} u_{\lambda}^{p^{*}}\right)^{\frac{\mu p}{p^{*}}}\right\},
$$

where $C$ is independent on $L$ and $\mathrm{C}$ is only dependent on $\Omega$ and $\left\|v_{\lambda}\right\|_{q}$. By the Fatou Lemma we have

$$
\left|u_{\lambda}\right|_{\mu p^{*}} \leq C^{\frac{1}{p \mu}} \mu^{\frac{1}{\mu}} \max \left\{\left|u_{\lambda}\right|_{p^{*}}, 1\right\} \leq C^{\frac{1}{\mu}} \mu^{\frac{1}{\mu}} \max \left\{1,\left|u_{\lambda}\right|_{p^{*}}\right\} .
$$

Similarly, let

$$
W_{L}=u_{\lambda} u_{L}^{\left(\mu^{2}-1\right)}, \quad Z_{L}=u_{\lambda} u_{L}^{p\left(\mu^{2}-1\right)}
$$

then we obtain

$$
\begin{aligned}
& \left(\int_{\Omega}\left|W_{L}\right|^{p^{*}}\right)^{\frac{p}{p^{*}}} \leq \\
& C \mu^{2 p} \int_{\Omega}\left(u_{\lambda}^{\mu^{2}+(p-1)\left(\mu^{2}-1\right)}+u_{\lambda}^{\mu^{2} p}+u_{\lambda}^{\mu^{2}+(p-1)\left(\mu^{2}-1\right)} v_{\lambda}^{\alpha}\right) .
\end{aligned}
$$

Using $\frac{p \mu}{p^{*}}+\frac{\alpha}{q^{*}}=1$ again gives

$$
\frac{\mu^{2}+(p-1)\left(\mu^{2}-1\right)}{p^{*} \mu}+\frac{\alpha}{q^{*}}<1 .
$$

From (4.21) and Hölder's inequality, it follows that

$$
\begin{aligned}
& \left(\int_{\Omega}\left|W_{L}\right|^{p^{*}}\right)^{\frac{p}{p^{*}}} \\
\leq & C \mu^{2 p}\left\{\left(\int_{\Omega} u_{\lambda}^{\mu p^{*}}\right)^{\frac{\mu^{2}+(p-1)\left(\mu^{2}-1\right)}{\mu p^{*}}}+\left(\int_{\Omega} u_{\lambda}^{\mu p^{*}}\right)^{\frac{\mu p}{p^{*}}}+\left(\int_{\Omega} u_{\lambda}^{\mu p^{*}}\right)^{\frac{\mu^{2}+(p-1)\left(\mu^{2}-1\right)}{\mu p^{*}}}\right\} .
\end{aligned}
$$

Due to the fact that

$$
\frac{\mu^{2}+(p-1)\left(\mu^{2}-1\right)}{\mu p^{*}} \leq \frac{\mu p}{p^{*}}
$$

we have

$$
\left(\int_{\Omega}\left|W_{L}\right|^{p^{*}}\right)^{\frac{p}{p^{*}}} \leq C \mu^{2 p} \max \left\{1,\left(\int_{\Omega} u_{\lambda}^{\mu p^{*}}\right)^{\frac{\mu p}{p^{*}}}\right\} .
$$

By virtue of the Fatou Lemma, we get

$$
\left|u_{\lambda}\right|_{\mu^{2} p^{*}} \leq C^{\frac{1}{\mu^{2}}} \mu^{\frac{2}{\mu^{2}}} \max \left\{1,\left|u_{\lambda}\right|_{\mu p^{*}}\right\} .
$$

Combining (4.20) and (4.22) yields

$$
\left|u_{\lambda}\right|_{\mu^{2} p^{*}} \leq C^{\frac{1}{\mu^{2}}} \mu^{\frac{2}{\mu^{2}}} \max \left\{\left|u_{\lambda}\right|_{\mu p^{*}}, 1\right\} \leq C^{\frac{1}{\mu}+\frac{1}{\mu^{2}}} \mu^{\frac{1}{\mu}+\frac{2}{\mu^{2}}} \max \left\{\left|u_{\lambda}\right|_{p^{*}}, 1\right\} .
$$


Repeating the above procedure, we have

$$
\left|u_{\lambda}\right|_{\mu^{k} p^{*}} \leq C^{\sum_{i=1}^{k} \mu^{-i}} \mu^{i=1} i^{k} \max \left\{\left|u_{\lambda}\right|_{p^{*}}, 1\right\} .
$$

By Proposition 6 and (4.23), we obtain a priori estimates of maximum norm of $\left\{u_{\lambda}\right\}$. Similarly, we can also derive a priori estimates for maximum norm of $\left\{v_{\lambda}\right\}$.

Using a similar argument as well as Young's inequality, we have

Proposition 8. Suppose that $\left(H_{1}\right)$ and $\left(H_{2}\right)$ hold. Then for any $\bar{\lambda}>0$, there exists $M>0$ such that

$$
\left|u_{\lambda}\right|_{\infty} \leq M, \quad\left|v_{\lambda}\right|_{\infty} \leq M
$$

hold for $\lambda \in[0, \bar{\lambda}]$ and each weak solution $\left(u_{\lambda}, v_{\lambda}\right)$ of system (1.1).

To prove the existence of the solution of system (1.1), we define the operator $\mathcal{M}$ such that if $\mathcal{M}(\lambda, w, z)=(u, v)$ if and only if

$$
\left\{\begin{aligned}
-\Delta_{p} u & =\lambda\left(a+|w|^{\delta}+|z|^{\alpha}\right), & & x \in \Omega, \\
-\Delta_{q} v & =\lambda\left(b+|w|^{\beta}+|z|^{\gamma}\right), & & x \in \Omega, \\
u=v & =0, & & x \in \partial \Omega .
\end{aligned}\right.
$$

It is clear that any nontrivial fixed point of $\mathcal{M}$ is the solution of system (1.1). Obviously, $\mathcal{M}$ is a completely continuous in $\mathbb{R}_{+} \times E_{0}$, so we can apply the LeraySchauder degree to study the existence of the solution of system (1.1).

Proof of Theorem 2. For any bounded and open $U$ with inner point $(0,0)$ in $E_{0}$, by the normal property of the Leray-Schauder degree we have

$$
\operatorname{deg}(\mathrm{I}-\mathcal{M}(0, \cdot, \cdot), \mathrm{U}, 0)=1 .
$$

Clearly, $\mathcal{M}(0, \cdot, \cdot)$ has a unique fixed point $(0,0)$. By Lemma 3 , it follows that $I-\mathcal{M}(\lambda, \cdot, \cdot)=0$ has a continuum $S_{+} \subset[0, \infty) \times E_{0}$ of solutions of system (1.1), and $S^{+}$is unbounded. Suppose that the projection of $S^{+}$in the positive direction of $\lambda$ is bounded, then the projection of $S^{+}$on the Banach space $E_{0}$ is unbounded. By Proposition 8 , it follows that when $\lambda$ lies on a bounded interval, and the corresponding solution-set must be bounded, which induces a contradiction. Consequently, $S^{+}$ is unbounded in the positive direction of $\lambda$. In virtue of connection of $S^{+}$, it follows that for any $\lambda>0$, the operator $\mathcal{M}(\lambda, \cdot, \cdot)$ has at least a fixed point.

Proof of Theorem 3. For any bounded and open $U$ with inner point $(0,0)$ in $E_{0}$, it follows that

$$
\operatorname{deg}(\mathrm{I}-\mathcal{M}(0, \cdot, \cdot), \mathrm{U}, 0)=1 .
$$

Apparently, $\mathcal{M}(0, \cdot, \cdot)$ has a unique fixed point $(0,0)$. By Lemma 3 it follows that $I-\mathcal{M}(\lambda, \cdot, \cdot)=0$ has a continuum $S^{+} \subset[0, \infty) \times E_{0}$ of solutions of system (1.1), and $S^{+}$is unbounded. By Proposition 4 , one can see that $S^{+}$is bounded in the positive $\lambda$-direction, so $S^{+}$has to become unbounded in the direction of the Banach space $E_{0}$. Let $S \subset[0, \infty) \times E_{0}$ be the set containing all solutions of $(u, v)=$ $\mathcal{M}(\lambda, u, v)$. In order to make $S^{+}$maximally connected, we replace $S^{+}$by the connected components of $S$ containing $S^{+}$. Without loss of generality, we still denote it by $S^{+}$. Let $\left[0, \lambda^{*}\right]$ be the projection of $S^{+}$onto the $\lambda$-direction. By the definition of $\lambda_{0}$, we have $\lambda^{*} \leq \lambda_{0}$. Since the prejection of $S^{+}$on the Banach space $E_{0}$ is unbounded, by Proposition 7 we see that it is impossible for $\lambda^{*}<\lambda^{0}$. Hence, we have $\lambda_{0}=\lambda^{*}$. Using Proposition 7 again, we deduce that there exist a sequence 
$\left\{\lambda_{n}\right\}$ and a corresponding sequence solutions $\left\{\left(u_{\lambda_{n}}, v_{\lambda_{n}}\right)\right\}$ of system (1.1) such that $\lambda_{n} \rightarrow \lambda^{*}$ and $\left|u_{\lambda_{n}}\right|_{\infty}+\left|v_{\lambda_{n}}\right|_{\infty} \rightarrow \infty($ as $n \rightarrow \infty)$.

\section{Acknowledgments}

The first author would like to thank Department of Mathematics of the University of Texas-Pan American for its hospitality and generous support during his visiting from June 2012 to October 2012.

\section{References}

[1] H. Amann, Fixed point equations and nonlinear eigenvalue problems in ordered Banach spaces, SIAM Rev., 18 (1976), 620-709.

[2] C. Azizieh, P. Clément and E. Mitidieri, Existence and a priori estimates for positive solutions of $p$-Laplace systems, J. Differential Equations, 184 (2002), 422-442.

[3] C. Bandle and W. Reichel, Solutions of quasilinear second order elliptic boundary value problem via degree theory, in: M. Chipot, P. Quittner (Eds.), Handbook of Differential Equations: Stationary Partial Differential Equations, Vol. 1, Elseriver, North-Holland, Amsterdam, 2004, pp. $1-70$.

[4] M. Benalili, Existence and multiplicity of solutions to elliptic equations of fourth order on compact manifolds, Dyn. Partial Differ. Equ. 6 (2009), 203-225.

[5] P. Clément, J. Fleckinger, E. Mitidieri, F. De Thélin, Existence of positive solutions for a nonvariational quasilinear elliptic system, J. Differential Equations, 166 (2000), 455-477.

[6] M. Cuesta and P. Takač, A strong comparison principle for positive solutions of degenerate elliptic equations, Differential Integral Equations, 13 (2000), 721-746.

[7] D.G. De Figueiredo, J.F. Yang, Decay, symmetry and existence of solutions of semilinear elliptic systems, Nonlinear Analysis, 33 (1998), 211-234.

[8] K. Deimling, Nonlinear functional analysis, Springer, Berlin, 1985.

[9] Z. Feng, S.Z. Zheng and H.F. Lu, Green's function of nonlinear degenerate elliptic operators and its application to regularity, Differential Integral Equations, 21 (2008), 717-741.

[10] J. Fleckinger and W. Reichel, Global solution branches for p-Laplacian boundary value problems, Nonlinear Analysis, 62 (2005), 53-70.

[11] J. García-Melián and J. Sabina de Lis, Maximum and comparison principles for operators involving the $p$-Laplacian, J. Math. Anal. Appl. 218 (1998), 49-65.

[12] B. Gidas and J. Spruck, A priori bounds for positive solutions of nonlinear elliptic equations, Comm. Partial Differential Equations, 6 (1981), 883-901.

[13] D.D. Hai and H.Y. Wang, Nontrivial solutions for $p$-Laplacian systems, J. Math. Anal. Appl. 330 (2007), 186-197.

[14] L. Iturriaga, S. Lorca and J. Sánchez, Existence and multiplicity results for the $p$-Laplacian with a $p$-gradient term, Nonlinear Differ. Equ. Appl. 15 (2008), 729-743.

[15] L. Montoro, B. Sciunzi and M. Squassina, Symmetry results for nonvariational quasi-linear elliptic systems, Advanced Nonlinear Studies, 10 (2010), 939-955.

[16] E. Mitidieri and S.I. Pohozaev, Nonexistence of positive solutions for quasilinear elliptic problems on $\mathcal{R}^{N}$, Proc. Steklov Inst. Math. 227 (1999), 186-216.

[17] A.D. Polyanin and V.F. Zaitsev, Handbook of Nonlinear Partial Differential Equations, 2nd Edition, Chapman and Hall/CRC, Florida, 2011.

[18] P. Tolksdorf, Regularity for a more general class of quasilinear elliptic equations, J. Differential Equations, 51 (1984), 126-150.

[19] H.Y. Wang, Existence and nonexistence of positive radial solutions for quasilinear systems, Suppliment of Discrete Contin. Dyn. Syst. 810-817, 2009.

[20] S.Z. Zheng and Z. Feng, Regularity for quasilinear elliptic systems with discontinuous coefficients, Dyn. Partial Differ. Equ. 5 (2008), 87-99.

[21] S.Z. Zheng, X.L. Zheng and Z. Feng, Regularity for a class of degenerate elliptic equations with discontinuous coefficients under natural growth, J. Math. Anal. Appl. 346 (2008), 359373 . 
School of Mathematical Science, Jiangsu Normal University, Xuzhou 221116, China

Department of Mathematics, University of Texas-Pan American, Edinburg, TX 78539, USA

E-mail address: zsfeng@utpa.edu 